\title{
Infants' sensitivity to the depth cue of shading
}

\author{
CARL E. GRANRUD \\ Carnegie-Mellon University, Pittsburgh, Pennsylvania \\ and \\ ALBERT YONAS and ELIZABETH A. OPLAND \\ University of Minnesota, Minneapolis, Minnesota
}

\begin{abstract}
Five- and 7-month-old infants were tested for sensitivity to the depth cue of shading. Infants were presented with two displays: a surface in which a convexity and a concavity were molded and a photograph in which shading specified a convexity and a concavity. Each display was presented under both monocular and binocular viewing conditions. Reaching was observed as the dependent measure. Infants in both age groups reached preferentially for the actual convexity in both the monocular and binocular viewing conditions. In the monocular photograph condition, the 7-month-olds reached preferentially for the apparent convexity specified by shading, indicating that they perceived it to be an actual convexity. These infants showed no significant reaching preference in the binocular photograph condition. This finding rules out interpretations of the infants' reaching not based on perceived depth. The results therefore suggest that the 7 month-olds perceived depth from shading. The 5-month-olds showed no significant reaching preferences when viewing the photograph; thus, they showed no evidence of depth perception from shading. These findings are consistent with the results of a number of studies that have investigated infants' sensitivity to pictorial depth cues. Together, these studies suggest that the ability to perceive depth from pictorial cues may first develop between 5 and 7 months of age.
\end{abstract}

Shading, variation in luminance in the retinal image, provides an effective source of visual information for the three-dimensional shapes of objects. Shading can create compelling impressions of three-dimensionality in paintings and photographs, and can specify the shapes of objects in natural environments. Shading provides information for three-dimensional shape because of the lawful relationship that exists between the orientation of a surface and luminance in the retinal image projected by the surface (Gibson, 1950). A surface will reflect more light when it is oriented orthogonally to the source of illumination than when it is oblique to the incident light. As a result, if illumination does not vary and if a surface is uniform in reflectance, gradients of luminance in the retinal image correspond to changes in the surface's orientation and, therefore, can specify three-dimensional object shape.

Perceiving shape from shading, however, involves a formidable problem for the visual system. Shading is potentially ambiguous because information for shape, or surface orientation, is confounded in the luminance values in the retinal image. The luminance of any point in the

This research was supported by National Institute of Child Health and Human Development Grants HD-05027 and R01-HD-16924-01. The authors thank Bill Merriman for his helpful comments on the manuscript and Martha Arterberry for her assistance in conducting the study. Correspondence concerning the article and requests for reprints should be sent to Carl E. Granrud, Department of Psychology, Carnegie-Mellon University, Pittsburgh, PA 15213. projected image of a surface is determined primarily by three variables: intensity of illumination, surface reflectance, and surface orientation. Consequently, a given luminance value at a point in the retinal image could result from an infinite number of combinations of these variables, and a gradient of luminance change could result from a change in any or all of these variables (Barrow \& Tenenbaum, 1978). Given this ambiguity, shading alone provides no information for direction of curvature in Figure 1. Interestingly, although shading is the only depth information in Figure 1, the figure is not normally seen as ambiguous. Generally, the form that is shaded on the bottom is perceived as convex and the form that is shaded on the top is perceived as concave. If the figure is inverted, the relief of each form reverses. The visual system appears to constrain the interpretations of shading through a process analogous to making assumptions about the scene that is being perceived. Most adult observers aparently assume implicity that illumination intensity and surface reflectance are approximately uniform and that there is a single light source located above the picture. Consequently, a convexity and a concavity are perceived.

The 19th century physicist David Brewster (cited in Gregory, 1966) argued that the assumption that light comes from above is learned through experience. If this hypothesis is correct, responsiveness to the depth cue of shading may appear late in development. There is now evidence, however, that, in one nonhuman species, responsiveness to shading is innate. Hershberger (1970) raised chicks in cages in which light always came from 


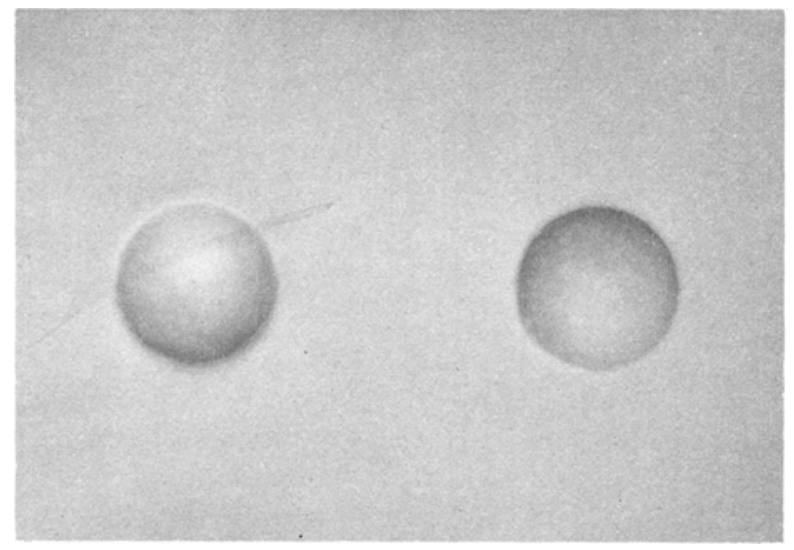

Figure 1. Convexity and concavity depicted by shading.

below. In a Skinner box with nondirectional illumination, the chicks were trained to peck at either a convexity or a concavity. After training, the chicks were presented with a display, similar to Figure 1, in which shading specified a convexity and a concavity. Chicks trained to peck at a convexity pecked reliably at the apparent convexity specified by shading; chicks trained to peck at a concavity pecked reliably at the apparent concavity. These findings demonstrate that chicks perceive direction of curvature from shading and that, for them, the "assumptions" that light comes from above and that illumination and reflectance are approximately uniform across a single surface are innate and uninfluenced by experience.

In the first study to investigate the development of depth perception from shading in humans, Fieandt (1938) tested six children with ages ranging from 4 to 7 years. He found some indication that these children had sensitivity to shading similar to that of adults. A clearer demonstration of children's depth perception from shading was provided by Benson and Yonas (1973). They presented 3-, 5- and 7-year-olds with a surface containing a convexity and a concavity, and trained them to point to the convexity. They then showed the children the photograph shown in Figure 1. Children in all three age groups exhibited transfer of training to the photograph, pointing consistently to the apparent convexity, the form that is shaded on the bottom. Thus, by at least 3 years of age, children can perceive direction of curvature from the depth cue of shading. In a subsequent study, Yonas, Kuskowski, and Sternfels (1979) found no increase in sensitivity to shading between the ages of 3 and 8 years when judgments of depth were based on the retinal frame of reference; sensitivity to depth information carried by the orientation of shading relative to the environment and to the location of a light source, however, improved considerably between the ages of 3 and 8 years.

Can infants perceive depth from shading? If the assumption that light comes from above must be learned from extensive experience with light and shadows, as Brewster suggested, infants may not have this ability. On the other hand, based on Hershberger's (1970) findings, it is conceivable that sensitivity to shading is present very early in life, or even at birth. The present study investigated human infants' sensitivity to the depth cue of shading. Five- and 7-month-old infants were presented with a surface in which a convexity and concavity were molded and a photograph in which shading specified an apparent convexity and concavity (Figure 1). Based on previous findings that 5- and 7-month-old infants reach preferentially for the nearer of two objects (Granrud, Yonas, \& Pettersen, 1984), it was hypothesized that infants would reach more frequently for the actual convexity than for the actual concavity. If infants reach preferentially for the actual convexity, infants who can perceive depth from shading should reach preferentially for the apparent convexity specified by shading in the photograph. In order to control for the possibility that infants might respond to nonspatial features in the photograph rather than to perceived depth or direction of curvature, the displays were presented under both monocular and binocular viewing conditions. If infants prefer to reach for the apparent convexity in the photograph not because it is perceived to be a convexity but because it is, for example, more highly illuminated on top, they should show similar reaching preferences in the monocular and binocular viewing conditions. If the infants' reaching is based on perceived depth, however, a stronger reaching preference should occur in the monocular condition than in the binocular condition, because binocular viewing should provide information about the flatness of the photograph.

\section{METHOD}

\section{Subjects}

Infant subjects were recruited from birth announcements published in the local newspapers. Fifty-three full-term infants served as subjects in the study: 24 7-month-olds (11 males, 13 females; mean age, 207.7 days; range, 199 to 219 days) and 295 -month-olds (13 males, 16 females, mean age, 150.1 days; range, 141 to 157 days). An additional 147 month-olds and 165 -month-olds were excluded from the sample because of state or attention problems, experimenter error, or failure to meet the criterion number of reaches ( 3 reaches in each condition).

\section{Apparatus}

The infants were presented with three displays (one at a time). One display, the three-dimensional display, was a gray matte-finished metal surface $(24 \times 35.5 \mathrm{~cm})$ into which were molded a convexity and a concavity $(10 \mathrm{~cm}$ apart, each $9 \mathrm{~cm}$ in diameter and $1 \mathrm{~cm}$ deep). The other two displays, the photograph displays (see Figure 1), were matte finished, rigidly mounted photographs $(30 \times 35.5 \mathrm{~cm})$ of a surface containing a convexity and a concavity $(8 \mathrm{~cm}$ apart, each $5 \mathrm{~cm}$ in diameter). The two photographs were mirror images of each other. The apparent convexity was on the right in one photograph and on the left in the other. A rod attached to the top edge of each display was used to bring the display toward the infant. The displays were presented in front of a homogeneous white background, and were illuminated from above by fluorescent ceiling lights and from the left side by a shaded $100-\mathrm{W}$ incandescent lamp $1.5 \mathrm{~m}$ away. Each experimental session was recorded on videotape with a television camera positioned behind the infant and approximately $45^{\circ}$ to the infant's left.

\section{Procedure}

There were four conditions in the experiment: threedimensional/monocular, three-dimensional/binocular, photo- 
graph/monocular, and photograph/binocular. Each infant was tested in all four conditions. The initial viewing condition (monocular or binocular) was chosen randomly. After 8 trials were completed in the initial viewing condition, 16 trials were presented in the other viewing condition. If the infant remained attentive after completing these trials, 8 more trials were presented in the initial viewing condition. In the monocular viewing condition, the infant wore a child-sized adhesive eyepatch over one eye (chosen randomly). The displays (three-dimensional and photograph) were presented in alternating pairs of trials; that is, two three-dimensional trials were followed by two photograph trials. The initial display was chosen randomly. The left-right position of the convexity was alternated on successive trials.

During the experiment, the infant sat on the parent's lap. The parent was instructed to hold the infant around the waist, leaving both of the infant's hands free for reaching. Prior to the beginning of each trial, the experimenter held the display approximately $60 \mathrm{~cm}$ directly in front of the infant and attempted to draw the infant's attention to the display. If necessary, the experimenter moved the display laterally until it was fixated by the infant. The experimenter then moved the display toward the infant until it was within reach, approximately $20 \mathrm{~cm}$ from the infant's eyes. The trial was terminated and the display was removed from the infant's view after the infant first touched either the convexity or the concavity, or touched both regions simultaneously with two hands, while attending to the display. The location of the reach was then scored by the experimenter and a new trial was initiated. If the infant reached without attending to the display or reached to a region of the display other than the convexity or concavity, the experimenter briefly moved the display back to the starting position and moved it toward the infant again. If approximately $30 \mathrm{sec}$ elapsed without a reach for the convexity or concavity, the trial was terminated. Trials were continued until 32 had been completed or until the infant stopped reaching or became fussy. Only infants who reached at least three times in each condition were included in the sample.

Each infant's reaching was scored by the experimenter during the session and again from the videotape record. The data scored from the videotape record were used in the analysis, unless an infant's reach was occluded on the video record, in which case live scoring was used. The region, convexity or concavity, first contacted by the infant's hand in each trial was scored. A second observer, who was unfamiliar with the hypotheses of the experiment, scored the reaching of eight randomly chosen 5-month-olds and seven randomly chosen 7-month-olds. Interjudge agreement, calculated using the $\mathrm{K}$ statistic (Bartko \& Carpenter, 1976), was $K=.90$ for data from the 7 -month-olds and $K=.85$ for data from the 5-month-olds.

\section{RESULTS AND DISCUSSION}

Table 1 shows the mean number of reaches scored in each condition and the mean percentages of the infants' reaches that first contacted the convexity or apparent convexity. Trials in which infants reached simultaneously for both the convexity and the concavity were excluded from

\section{Table 1}

Mean Number of Reaches Scored and Mean Percentage of Reaches to Convexity

\begin{tabular}{|c|c|c|c|c|c|c|c|}
\hline & \multicolumn{3}{|c|}{$\begin{array}{c}\text { Mean number } \\
\text { of reaches }\end{array}$} & \multicolumn{4}{|c|}{$\begin{array}{l}\text { Mean percentage of } \\
\text { reaches to convexity }\end{array}$} \\
\hline & Mo & nocular & Binocular & Mol & ocular & Bin & locular \\
\hline \multicolumn{8}{|c|}{ Three-Dimensional Condition } \\
\hline $\begin{array}{l}\text { 7-month-olds } \\
\text { 5-month-olds }\end{array}$ & $\begin{array}{l}5.6 \\
4.6\end{array}$ & $\begin{array}{l}(1.7) \\
(1.4)\end{array}$ & $\begin{array}{ll}6.1 & (1.7) \\
4.7 & (1.5)\end{array}$ & $\begin{array}{l}64.4 \\
62.7\end{array}$ & $\begin{array}{l}(18.0) \\
(19.2)\end{array}$ & $\begin{array}{l}74.2 \\
60.1\end{array}$ & $\begin{array}{l}(16.0) \\
(18.7)\end{array}$ \\
\hline \multicolumn{8}{|c|}{ Photograph Condition } \\
\hline $\begin{array}{l}\text { 7-month-olds } \\
\text { 5-month-olds }\end{array}$ & $\begin{array}{l}5.7 \\
4.6\end{array}$ & $\begin{array}{l}(2.0) \\
(1.3)\end{array}$ & $\begin{array}{ll}6.1 & (1.6) \\
4.2 & (1.3)\end{array}$ & $\begin{array}{l}62.8 \\
53.8\end{array}$ & $\begin{array}{l}(14.7) \\
(18.5)\end{array}$ & $\begin{array}{l}51.4 \\
50.0\end{array}$ & $\begin{array}{l}(15.2) \\
(19.8)\end{array}$ \\
\hline
\end{tabular}

Note-Standard deviations are given in parentheses. the data. As a result, the percentage of reaches to the convexity plus the percentage of reaches to the concavity equaled 100.

The data from the three-dimensional and photograph conditions were analyzed separately, because these conditions asked two distinct questions. The threedimensional conditions asked whether infants would reach more frequently for a convexity than for a concavity. The goal of these conditions was to determine whether reaching is a valid measure for studying infants' depth perception from shading. The photograph conditions asked whether infants could perceive depth, or direction of surface curvature, from shading. Separate analyses were performed to address each of these questions directly.

The infants' reaching preferences in the threedimensional conditions were analyzed in a $2 \times 2$ analysis of variance with repeated measures, with age (5 and 7 months) as a between-subjects factor and viewing condition (monocular and binocular) as a within-subjects factor. The dependent variable was the percentage of reaches scored as contacting the convexity. This analysis revealed a significant main effect for age $[F(1,51)=5.04$, $\mathrm{p}<.05]$. No other effect reached statistical significance. The main effect for age indicates that overall, the 7-montholds reached more consistently for the convexity than did the 5-month-olds. Of primary interest, however, was whether the infants' reaching preferences for the convexity differed significantly from chance performance. Four planned comparisons, using the least significant difference (LSD) procedure (Kirk, 1982), were performed to address this issue $(\alpha=.05)$. These comparisons revealed that both the 5- and 7-month-olds showed significant reaching preferences for the convexity in both viewing conditions. This finding suggests that if these infants can perceive depth from shading, they should reach preferentially for the apparent convexity specified by shading in the photograph displays.

The infants' reaching preferences in the photograph conditions were analyzed in a $2 \times 2$ analysis of variance with repeated measures, with age (5 and 7 months) as a between-subjects factor and viewing condition (monocular and binocular) as within-subjects factor. The dependent variable was the percentage of reaches scored as contacting the apparent convexity. This analysis revealed a significant main effect for viewing condition $[F(1,51)=$ $5.03, p<.05]$. No other effects were statistically significant. The main effect for viewing condition indicates that the infants' reaching preference for the convexity was significantly greater in the monocular condition than in the binocular condition. Although this finding suggests that infants can perceive depth from shading, it is not clear from this analysis that infants in both age groups showed sensitivity to shading. A set of planned comparisons, using the LSD procedure, was performed to analyze the data from each age group separately $(\alpha=.05)$. The 7-montholds showed a significant reaching preference for the apparent convexity in the monocular condition but not in the binocular condition. In addition, the 7-month-olds' 
reaching preference was significantly greater in the monocular condition than in the binocular condition. This finding indicates that the 7-month-olds' reaching preference in the monocular condition was based on perceived depth, and cannot be attributed to nonspatial features in the display; if infants reached for the apparent convexity not because they perceived it to be a convexity but because it was more highly illuminated on top, for example, they should have shown equivalent reaching preferences in the monocular and binocular viewing conditions. The results therefore indicate that the 7-month-olds perceived depth from shading.

In contrast to the 7-month-olds, the 5-month-olds showed no significant reaching preference in either viewing condition and their performances did not differ significantly between the monocular and binocular viewing conditions. The 5-month-olds thus showed no evidence of depth perception from shading. Based on the results of the three-dimensional conditions indicating that 5month-olds reach consistently for a convexity when they perceive it as such (i.e., when additional information is available, such as motion parallax and accommodation in the monocular condition, plus binocular disparity and convergence in the binocular condition), it seems likely that the 5-month-olds did not perceive a convexity from shading in the photograph. However, we cannot be certain from these results that 5-month-olds are insensitive to the depth cue of shading. Motion parallax information specifying the flatness of the photographs was present in the monocular/photograph condition. It is possible that 5-month-olds can perceive depth from shading but that motion parallax is a more effective depth cue than shading for infants of this age. As a result, the 5-month-olds may have responded to motion parallax that specified the flatness of the photographs rather than to shading that specified the presence of a convexity and a concavity. Perhaps a more sensitive test of 5-month-old infants' depth perception from shading could be achieved if conflicting motion parallax could be minimized.

Although we cannot be certain that 5-month-old infants are incapable of perceiving depth from shading, the results of this study are consistent with the developmental pattern found in a number of previous studies that have investigated infants' depth perception from pictorial depth cues. Seven-month-old infants perceive the spatial layout depicted in Ames's (1951) trapezoidal window illusion, an illusion created by several pictorial depth cues (Kaufmann, Maland, \& Yonas, 1981; Yonas, Cleaves, \& Pettersen, 1978), and they respond appropriately to the depth cues of familiar size (Granrud, Haake, \& Yonas, 1985; Yonas, Pettersen, \& Granrud, 1982), relative size (Yonas, Granrud, \& Pettersen, 1985), and interposition (Granrud $\&$ Yonas, 1984). Five-month-olds, in contrast, have not been found to be sensitive to any of these depth cues (for a review of these studies, see Yonas \& Granrud, 1985). The consistent finding that 7-month-olds are sensitive to pictorial depth cues and the consistent failure to find sensitivity in 5-month-olds suggests that the ability to per- ceive depth from pictorial cues may first develop between 5 and 7 months of age. These findings further suggest that infants may become sensitive to several pictorial depth cues simultaneously. If future research confirms that there is simultaneous emergence of sensitivity to different pictorial depth cues, it would have an important implication regarding the mechanism underlying the development of depth perception from pictorial cues. If the development of sensitivity to each different depth cue required a specific type of learning or experience, or if sensitivity to each cue was achieved by a distinct mechanism, we might expect the appearance of sensitivity to various cues to be not simultaneous, but staggered. Simultaneous emergence of sensitivity to several different pictorial depth cues might, therefore, suggest that a common mechanism, which matures sometime between 5 and 7 months of age, is required for depth perception from these cues.

The finding that 7-month-old infants can perceive depth from shading suggests that early in life the visual system constrains the interpretation of variation in retinal luminance by exploiting several stable properties of the terrestrial environment. As noted above, information for surface orientation is confounded in retinal luminance because the luminance of any point in the image of a surface is jointly determined by several variables, including direction and intensity of illumination, surface reflectance, and surface orientation. Barrow and Tenenbaum's (1978) theoretical analysis of how shape could be detected from shading suggests that, to recover information for shape from image luminance, the visual system must make assumptions about the world and exploit the constraints these assumptions imply. The 7-month-old infant's visual system appears to constrain the possible interpretations of image luminance by incorporating into its functioning processes analogous to assumptions that illumination and reflectance are approximately uniform across a single surface. As a result, smooth variations in luminance are perceived as due to changes in surface orientation. Although these assumptions resulted in innacurate perception in the present study (shading in the photographs was actually caused by changes in reflectance, not in surface orientation), they probably lead to veridical perception in natural environments, because they reflect enduring properties of light and surfaces in the environment to which the visual system is adapted.

We must emphasize that it remains unknown how assumptions about illumination and surface reflectance are embodied in the visual system. We do not mean to imply that these assumptions are explicitly represented; they may be instantiated in the functioning of a mechanism that makes no explicit assumptions or computations (e.g., see Runeson, 1977). However, there is no obvious way to explain the results of this study without positing perceptual processes that, at some level, are analogous to making the assumptions that illumination and reflectance are uniform across a single surface. The finding that infants can perceive depth from shading suggests that these assumptions are not based on conscious thinking or ad- 
vanced cognition. Instead, they appear to be automatic rules for extracting depth information from retinal luminance. Perhaps these rules are innate in humans as well as in newborn chickens (Hershberger, 1970). On the other hand, the finding that 5-month-old infants may be unable to perceive depth from shading leaves open the possibility that learning is involved in the development of this aspect of spatial perception.

\section{REFERENCES}

AmEs, A., JR. (1951). Visual perception and the rotating trapezoidal window. Psychological Monographs, 65(Whole No. 324).

Barrow, H. G., \& Tenenbaum, J. M. (1978). Recovering intrinsic scene characteristics from images. In A. R. Hanson \& E. M. Riseman (Eds.), Computer vision systems. New York: Academic Press.

BartKo, J. J., \& CARPENTER, M. D., JR. (1976). On the methods and theory of reliability. Journal of Nervous and Mental Disease, 163, 307-317.

Benson, K. A., \& Yonas, A. (1973). Development of sensitivity to static pictorial depth information. Perception \& Psychophysics, 13, 361-366.

FIEANDT, K., voN (1938). Über Sehen von Tiefengebilden bei Wechselnder Beleuchtungsrichtung (Unpublished manuscript). Helsinki: University of Helsinki, Department of Psychology.

Gibson, J. J. (1950). The perception of the visual world. Boston: Houghton-Mifflin.

Granrud, C. E., HAAKe, R. J., \& Yonas, A. (1985). Infants' sensitivity to familiar size: The effect of memory on spatial perception. Perception \& Psychophysics, 37, 459-466.

Granrud, C. E., \& Yonas, A. (1984). Infants' perception of pictorially specified interposition. Journal of Experimental Child Psychology, 37, 500-511.
Granrud, C. E., Yonas, A., \& Pettersen, L. (1984). A comparison of monocular and binocular depth perception in five- and seven-monthold infants. Journal of Experimental Child Psychology, 38, 19-32.

GREGORY, R. L. (1966). Eye and brain: The psychology of seeing. New York: McGraw-Hill.

HERShBERGER, W. (1970). Attached-shadow orientation perceived as depth by chickens reared in an environment illuminated from below. Journal of Comparative and Physiological Psychology, 73, 407-411.

Kaufmann, R., Maland, J., \& Yonas, A. (1981). Sensitivity of 5and 7-month-old infants to pictorial depth information. Journal of Experimental Child Psychology, 32, 162-168.

KIRK, R. E. (1982). Experimental design: Procedures for the behavioral sciences. Monterey, CA: Brooks/Cole.

RUNESON, S. (1977). On the possibility of 'smart' perceptual mechanisms. Scandinavian Journal of Psychology, 18, 172-179.

Yonas, A., Cleaves, W., Pettersen, L. (1978). Development of sensitivity to pictorial depth. Science, 200, 77-79.

Yonas, A., \& GranRud, C. E. (1985). The development of sensitivity to kinetic, binocular, and pictorial depth information in human infants. In D. Ingle, D. Lee \& M. Jeannnerod (Eds.), Brain mechanisms and spatial vision (pp. 113-145). Dordrect, Netherlands: Martinus Nijof.

Yonas, A., Granrud, C. E., \& Pettersen, L. (1985). Infants' sensitivity to relative size information for distance. Developmental Psychology, 21, 161-167.

Yonas, A., Kuskowski, M., \& Sternfels, S. (1979). The role of frames of reference in the development of responsiveness to shading information. Child Development, 50, 495-500.

Yonas, A., Pettersen, L., \& Granrud, C. E. (1982). Infants' sensitivity to familiar size as information for distance. Child Development, 53, $1285-1290$.

(Manuscript received May 17, 1984; revision accepted for publication March 18, 1985.) 